\title{
Macroscopic Chirality of Twist-Bend Nematic Phase in Bent Dimers Confirmed by Circular Dichroism
}

\author{
Warren D. Stevenson ${ }^{\mathrm{a}}$, Xiangbing Zeng*a ${ }^{*}$, Chris Welch ${ }^{\mathrm{b}}$, Anil K. Thakur ${ }^{\mathrm{a}}$, Goran Ungar*a,c and Georg \\ H. Mehl ${ }^{\mathrm{b}}$ \\ a Department of Materials Science and Engineering, University of Sheffield, Sheffield S1 3JD, UK \\ ${ }^{\mathrm{b}}$ Department of Chemistry, University of Hull, Hull HU6 7RX, UK \\ ${ }^{\mathrm{c}}$ State Key Laboratory for Mechanical Behavior of Materials, School of Material Science and \\ Engineering, Xian Jiaotong University, Xi'an 710049, P.R. China
}

\begin{abstract}
Many achiral bent molecules and some polymers with such repeat units exhibit a liquid crystal phase transition between a conventional nematic $(\mathrm{N})$, and a second nematic $\left(\mathrm{N}_{\mathrm{x}}\right)$ with periodically modulated orientation. Theory predicts several possible structures for the $\mathrm{N}_{\mathrm{x}}$ phase, some of which are chiral (helical), and others achiral (e.g. zigzag). Experimentally distinguishing which modulation type is present is non-trivial and the structure is often assumed to be helical despite the other possibilities. Here we use circular dichroism (CD) spectroscopy to directly and unambiguously identify chirality in the $\mathrm{N}_{\mathrm{x}}$ phase of two different dimer series, "DTC5Cn" and "CBCnCB", which vary in $\left(\mathrm{CH}_{2}\right)_{\mathrm{n}}$ spacer length $(n=5,7,9)$. In doing so we have determined that the modulation type is helical in all samples. The temperature dependence of the CD signal was also investigated, suggesting that the helical structure progressively unwinds on approach to the high temperature $\mathrm{N}$ phase.
\end{abstract}

\section{Introduction}

A thermotropic nematic-nematic liquid crystal (LC) phase transition was first experimentally observed in the 1990's in a series of main-chain copolyethers, ${ }^{1-3}$ then later in bent dimers, ${ }^{4-9}$ rigid bent-core molecules, ${ }^{10}$ and more recently in trimers ${ }^{11,12}$ and larger oligomers. ${ }^{13-16}$ In all instances (excluding the rigid core compound) connected mesogens were angled to each other, most commonly by odd numbered alkylene spacers. In reports of nematic-nematic transitions, the high- $T$ phase is found to be the conventional uniaxial nematic $(\mathrm{N})$, where molecules lack positional correlation but preferentially align their longest axis parallel to each other, defining a locally linear director field with cylindrical symmetry. On transition to the low- $T$ phase, the linear director field becomes orientationally modulated, reducing molecular-scale phase symmetry. In a popular theoretical study by Dozov, ${ }^{17}$ two thermodynamically stable nematic modulations are predicted to occur with equal probability. These are twist-bend nematic $\left(\mathrm{N}_{\mathrm{tb}}\right)$ and splay-bend nematic $\left(\mathrm{N}_{\mathrm{sb}}\right)$. We also note the polar-twisted nematic $\left(\mathrm{N}_{\mathrm{pt}}\right)$ model by Vanakaras and Photinos, ${ }^{18}$ but group it with the $\mathrm{N}_{\mathrm{tb}}$ phase due to its structural similarity. In the $\mathrm{N}_{\mathrm{tb}}$ model, achiral molecules form a helical structure where mesogens tilt in respect to the helical axis (Figure 1a). Rather than chemical chirality, the $\mathrm{N}_{\mathrm{tb}}$ helix is stabilized by local twist and bend elastic distortions created by close-packing of curved conformations. ${ }^{17}$ It could also be stabilized by polar interactions. ${ }^{18}$ In the simplest alternative $\mathrm{N}_{\mathrm{sb}}$ model, bent molecules periodically flip orientation, resulting in an achiral 'wave' or 'zig-zagging' director field, rather than a helix (Figure 1b). ${ }^{17}$ The key experimental findings in support of the $\mathrm{N}_{\mathrm{tb}}$ model are periodic striations in freezefracture TEM images, ${ }^{5,6}$ AFM investigations, ${ }^{19}$ resonant X-ray diffraction peaks arising from a 8-12nm orientational period $^{20-22}$ and the appearance of an additional quadrupolar doublet in NMR spectra. ${ }^{9,23-24}$ The additional doublet is considered to arise from symmetry breaking on transition from the $\mathrm{N}$ phase. However, orientational periodicity and reduced nano-scale symmetry would also arise in the $\mathrm{N}_{\mathrm{sb}}$ phase. Simulations by Salamończyk et al. ${ }^{22}$ suggest the $\mathrm{N}_{\mathrm{sb}}$ structure should also produce resonant X-ray diffraction peaks. As the $\mathrm{N}_{\mathrm{tb}}$ and $\mathrm{N}_{\mathrm{sb}}$ have very similar local structures, the ability to determine long- 
range phase chirality in the bulk low- $T$ phase is therefore of extreme importance when studying nematic-nematic transitions. This could be the only reliable way to unambiguously distinguish the $\mathrm{N}_{\mathrm{tb}}$ from the $\mathrm{N}_{\mathrm{sb}}$ phase.

One capable method that is yet to be reported in the context of the nematic-nematic transition is circular dichroism (CD). In CD spectroscopy the sample is periodically exposed to left and right circularly polarized light using a photoelastic modulator (PEM) and a polarizer. The PEM acts as an alternating $+\lambda / 4$ and $-\lambda / 4$ plate. The difference in the absorbance of the sample $\Delta A_{C D}=A_{L}-A_{R}$ is measured as a function of wavelength. If the chromophores do not absorb both polarisations equally, i.e. $\Delta \mathrm{A} \neq 0$, they must reside in a chiral environment, with relatively few exceptions. ${ }^{25-31}$ Because a difference in absorption coefficients of L- and R-light also converts linearly polarized light to slightly elliptically polarized (Figure S4), CD instruments normally express the signal in ellipticity $(\phi)$ units, where $\phi=\arctan (\mathrm{p} / \mathrm{q})$, with $\mathrm{p}$ and $\mathrm{q}$ being the short and the long axes of the ellipse. $\phi$, in millidegrees, equals $3.298 \times 10^{4} \Delta \mathrm{A}_{\mathrm{CD}}$. However, when the sample is also linearly dichroic (LD), a phenomenon called CD-LD crosstalk results in artefacts due to the linear signal, as a result of additional static phase shift due to residual strain in the PEM or other optical element. ${ }^{32} \mathrm{CD}$ is not generally used to study optically anisotropic materials, such as LCs, because in such samples LD is much stronger than CD, and is known to cause artefacts in CD measurements. One of the signs of the existence of such artefacts is dramatic variation in measured CD signals change, often even change of sign, when rotating the sample around the beam axis (some examples are given in Section 5.3, SI). These interfering effects must therefore be eliminated or at least minimised; the best way of achieving this is aligning the optic axis with the incident beam, so that the material acts as if it was optically isotropic. ${ }^{33}$

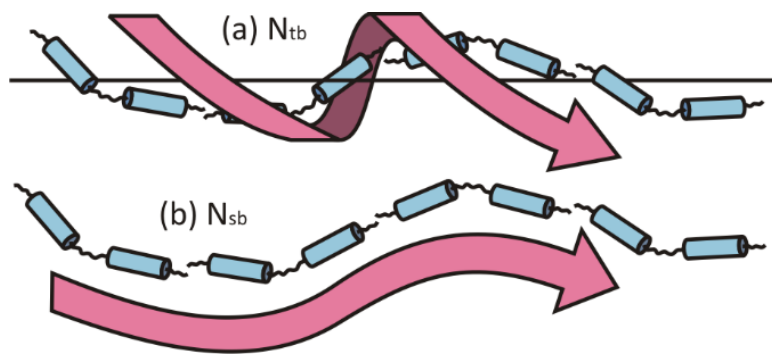

Figure 1 - Diagrams of the twist-bend $\mathrm{N}_{\mathrm{tb}}$ (a) and splay-bend $\mathrm{N}_{\mathrm{sb}}$ (b) nematics.

In this work we investigated the chirality of the low- $T$ nematic $\left(\mathrm{N}_{\mathrm{x}}\right)$ in two series of bimesogenic compounds: diterphenyls DTC5Cn $(n=5,7,9)$ and dicyanobiphenyls $\operatorname{CBCnCB}(n=7,9)$, where $n$ is the number of methylene groups in the spacer - see Figure 2a. These are two archetypal and moststudied series of $\mathrm{N}_{\mathrm{x}}$-forming bimesogens, so the results obtained here can be regarded as representative of bent flexibly-linked bimesogens. We succeeded in aligning the $\mathrm{N}_{\mathrm{x}}$ phase homeotropically by shearing, which allowed circularly polarised light to be directed along the optic axis, greatly minimising linear dichroism contribution to CD spectra. As a result we show conclusively that the lower temperature phase of all studied compounds is chiral, indicating that they are all of $\mathrm{N}_{\mathrm{tb}}$ type. Important conclusions were also drawn from the temperature dependence of the CD signal. Homeotropic alignment was investigated by polarized optical microscopy (POM) and grazing incidence X-ray scattering (GIXS).

\section{Results}

\section{Thermal Behavior}

The thermal behavior of DTC5Cn $(n=5,7,9)$ and $\operatorname{CBCnCB}(\mathrm{n}=7$ and 9$)$ has been studied previously. ${ }^{34,35}$ DTC5C5, 7 and 9 undergo the following phase sequence on cooling: 
Iso $\rightarrow \mathrm{N} \rightarrow \mathrm{N}_{\mathrm{x}} \rightarrow \mathrm{Sm} \rightarrow$ Cryst, where 'Iso', 'Sm' and 'Cryst' indicate isotropic, smectic and crystal phases, respectively. In the case of DTC5C7 and DTC5C9 the Sm phase is metastable and only seen on cooling (monotropic). $\mathrm{CBC7CB}$ and $\mathrm{CBC} 9 \mathrm{CB}$ all go directly from $\mathrm{N}_{\mathrm{x}}$ to Cryst. Relevant thermal data are displayed in Table 1.

Table 1 - DTC5Cn and CBCnCB Thermal Data on Heating/Cooling, taken from refs 34 (DTC5Cn) and 3535 (CBCnCB).

$T_{\text {shear }}$ is the shearing temperature

\begin{tabular}{|c|c|c|c|c|}
\hline Sample & $T_{\text {Cryst } / \mathrm{Nx}}\left({ }^{\circ} \mathrm{C}\right)$ & $T_{\mathrm{Sm} / \mathrm{Nx}}\left({ }^{\circ} \mathrm{C}\right)$ & $T_{\mathrm{Nx} / \mathrm{N}}\left({ }^{\circ} \mathrm{C}\right)$ & $T_{\text {Shear }}\left({ }^{\circ} \mathrm{C}\right)$ \\
\hline DTC5C5 & $-/-$ & $114.7 / 113.5$ & $121.6 / 120.5$ & 120 \\
\hline DTC5C7 & $98.6 /-$ & $-/ 93.4$ & $127.5 / 126.3$ & 110 \\
\hline DTC5C9 & $95.9 /-$ & $-/ 83.8$ & $127.9 / 126.9$ & 100 \\
\hline CBC7CB & $102.0 /-$ & $-/-$ & $104.4 / 103.0$ & 80 \\
\hline CBC9CB & $85.2 / 52.5$ & $-/-$ & $104.9 / 107.3$ & 100 \\
\hline
\end{tabular}

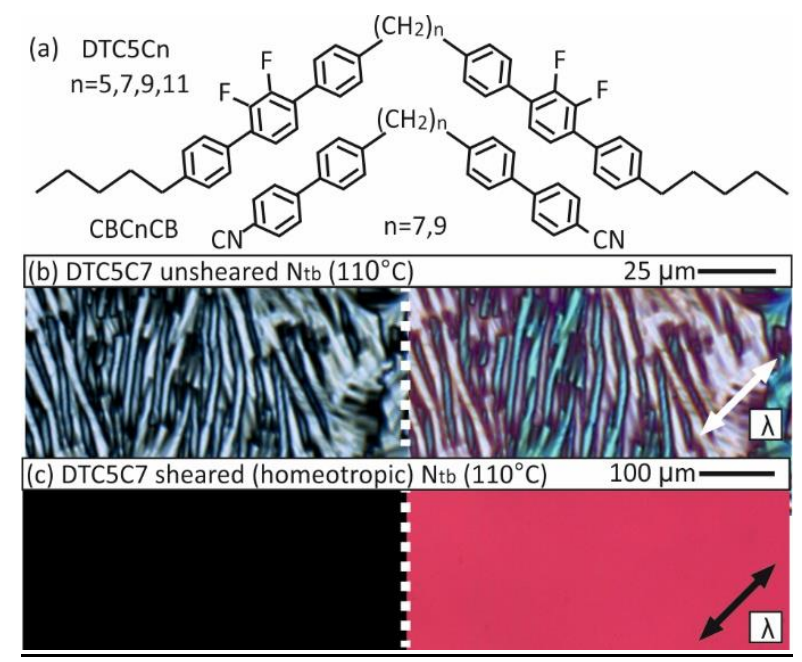

Figure 2 - (a) Molecular structures of DTC5Cn (top) and CBCnCB (bottom) dimers. (b) Rope-like $\mathrm{N}_{\mathrm{x}}$ texture in DTC5C7 obtained on cooling from the $\mathrm{N}$ phase. (c) Homeotropically aligned $\mathrm{N}_{x}$ phase in DTC5C7 after shearing at $110^{\circ} \mathrm{C}$. This can be achieved in all other compounds after shearing. (b) and (c) images on the right were taken with a $\lambda$-plate with the slow axis at $45^{\circ}$ to the polarisers, as indicated.

\section{Polarized Optical Microscopy (POM)}

The $\mathrm{N}-\mathrm{N}_{\mathrm{x}}$ transition appears similar in all six compounds: large domains of uniform texture or with Schlieren defects, marking the $\mathrm{N}$ phase, become striped or rope-like at the transition to the $\mathrm{N}_{\mathrm{x}}$ (Figure 2b). The stripes are explained as being an undulation pattern ${ }^{6}$ and their width has been shown to depend on cell thickness. ${ }^{36}$ The blue stripes in Figure $1 \mathrm{~b}$ (right) indicate that the director is aligned southwest-northeast, and yellow stripes having director aligned southeast-northwest. The effect of an applied shearing force on the $\mathrm{N}_{\mathrm{x}}$ phase was investigated. Depending on temperature, shearing in the $\mathrm{N}_{\mathrm{x}}$ phase resulted in a near perfect black field of view in all five samples (Figure 2c). The field of view remained black upon in-plane rotation, signifying homeotropic alignment of the $\mathrm{N}_{\mathrm{x}}$ phase, as also confirmed by the conoscopic image of the sample (Figure S1, SI). It is difficult to achieve defect free alignment in some samples, but the key is finding the correct temperature - Too high and the sample is too fluid, too low 2 nd it is too viscous or crystallizes. The temperature at which we were best able to achieve homeotropic alignment by shearing $\left(T_{\text {shear }}\right)$ is provided in Table 1 . All samples maintain the black field of view for over two hours in isothermal conditions. The black field of view is also maintained as the temperature is raised or lowered, but is lost at the onset of phase transitions where the field of view becomes textured and brighter. 


\section{Grazing Incidence X-Ray Scattering (GIXS)}

The nematic phases of the DTC5Cn $(n=5,7,9)$ compounds were investigated by GIXS on station BM28 of the ESRF, and that of DTC5C7 is shown in Figure 3. Similar GIXS patterns of CBC9CB are shown in Figure S2 (SI). In each case the sample was brought to the isotropic phase and cooled into the $\mathrm{N}$ and then $\mathrm{N}_{\mathrm{x}}$ phase. Without shearing the $\mathrm{N}$ and $\mathrm{N}_{\mathrm{x}}$ phases of the four samples mostly showed planar orientation (nematic director preferentially in-plane) on the silicon surface, evidenced by the azimuthal positions of the wide angle X-ray scattering (WAXS). In Figure 3 a the diffuse WAXS arc ( $q 3$ centered $\sim 13.8 \mathrm{~nm}^{-1}$, corresponding d-spacing $\sim 0.46 \mathrm{~nm}$ ), arising from side-by-side mesogen packing, is centred on the vertical axis, indicating that the molecules are mostly laying down on the horizontal substrate. Note that only half of the scattering pattern is visible by GIXS. While being held

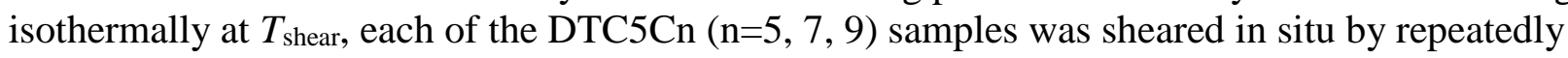
sliding a second heated silicon plate over the LC surface. Following this procedure homeotropic orientation of the phase was obtained (Figure 3b). The two diffuse small angle X-ray scattering (SAXS) peaks, $q 1$ centered at $\sim 1.57 \mathrm{~nm}^{-1}$ and $q 2$ at $3.30 \mathrm{~nm}^{-1}$, have corresponding d-spacings $4.0 \mathrm{~nm}$ and $1.9 \mathrm{~nm}$ respectively, using Bragg equation. These match roughly the full and half length $(4.5 \mathrm{~nm}$ and $2.25 \mathrm{~nm}$ ) of the DTC5C7 dimer. The two peaks arise from local layering of mesogens (cybotaxis ${ }^{37}$ ). They are centered on the surface normal with WAXS peaks $(q 3)$ on the horizon, indicating the local layer planes have a preferred orientation parallel to the silicon surface. The FWHM of $\mathrm{q} 1$ and $\mathrm{q} 2$ is $\sim 0.6 \mathrm{~nm}^{-1}$ in the vertical direction, indicating that the size of the local layers is $\sim 10 \mathrm{~nm}$. The presence of the " 1 st order" SAXS peak, its d-spacing 4.0nm matching that of the length of the dimer, also indicates that alkyl end-chains and spacers in DTC5Cn dimers do not mix, at least not completely. The presence of local layering also explains why shear produces homeotropic alignment as it does also in smectics but not in ordinary nematics. After heating back into the $\mathrm{N}$ phase, the samples returned to a mostly planar orientation as observed before shearing.

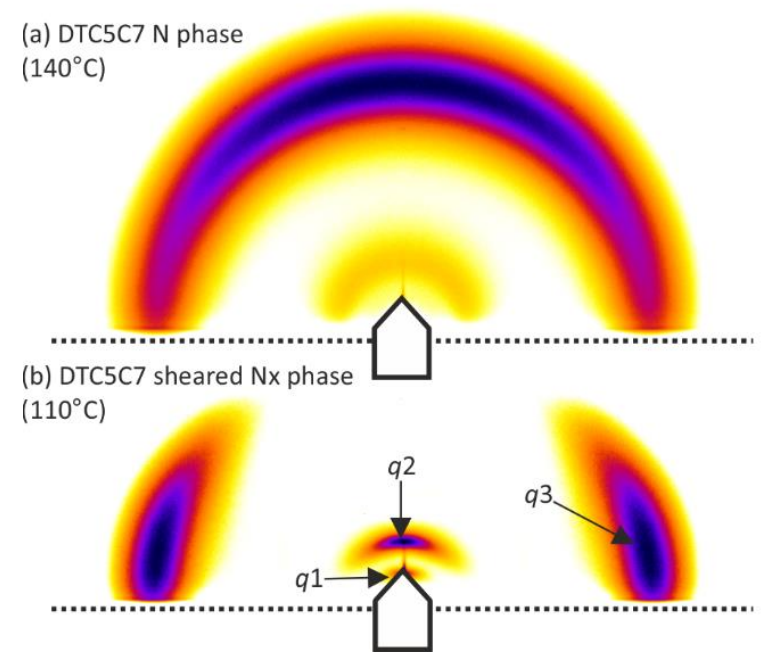

Figure 3 - Representative GIXS patterns of DTC5C7. q1-3 denote the scattered wave vectors corresponding to the scattering peaks. (a) N phase, un-sheared showing mostly planar orientation. (b) Homeotropic $\mathrm{N}_{\mathrm{x}}$ phase after shearing at $110^{\circ} \mathrm{C}$. In (b) the WAXS intensity (q3) has been increased by a factor of 2 compared to the SAXS for display purposes.

\section{Circular Dichroism (CD) and UV-Vis}

$\mathrm{CD}$ and UV-Vis experiments were conducted on station B23 of the Diamond Light Source. Inside the optical chamber, each sample was held in a quartz glass sandwich cell, resting on a rotatable Linkam hot stage. The rotatable stage was used to measure the CD spectrum in various sample orientations (every $45^{\circ}$ ) so that, if present, the artefacts due to linear dichroism of samples could be identified and reduced by averaging. The sample thickness was estimated in each case using absorption spectra, 
which were recorded simultaneously with CD (see SI). Thickness correction has been applied for comparison between different samples, as the measured CD signal is proportional to sample thickness.

After shearing, the CD spectra of all samples contain a peak in the region of 290-330 nm, attributed to absorption from $\pi$-conjugated parts of the molecule (Figure 4e). The spectrum of the CBC9CB (original CD peak positive, Figure S5a, SI) is inverted, and the CBCnCB spectra were divided by 2 for easier comparison with those of DTC5Cn samples, the CD peaks of which are much weaker. In the homeotropic $\mathrm{N}_{\mathrm{x}}$ phase, the sign of the CD peak is invariant with sample rotation about the axis of the light beam while its magnitude varies only slightly (examples Figures 4a, c and SI), showing that the signal originates from sample chirality and not LD effects - for a similar CD experiment but on chiral columnar phases see Nagayama et al. ${ }^{27}$ This confirms that all five samples are of $\mathrm{N}_{\mathrm{tb}}$ type - This notation will now be adopted in place of $\mathrm{N}_{\mathrm{x}}$.

Three different spots on each sample were investigated to check for consistency, but CD varied very little and the peak remained the same sign. The peak sign also stayed the same after quickly flipping the quartz cell upside down (Figure S6, SI). The sign and shape of a CD peak is linked to the details of intra- and inter-molecular chiral interactions (molecular conformation and packing) and how they affect the electronic states of the molecules. However, for a molecular level understanding of the CD spectrum computational studies of the electronic states of different $N_{\mathrm{tb}}$ forming dimers $e . g$. using density functional theory are needed, and may help differentiate different molecular models of the $\mathrm{N}_{\mathrm{tb}}$ phase. The apparent homochirality of our cells contradicts some polarized microscopy reports in planar aligned cells, where coexisting domains of opposite handedness have been reported. ${ }^{38,39}$ However, in such experiments artefacts (similar to discussed earlier for CD experiments), originating from sample birefringence and linear dichroism, cannot be easily excluded. Homochirality may be a consequence of the shearing process, which forces neighbouring domains to mix and effectively choose a single handedness.

In the planar oriented $\mathrm{N}$ phase the sign of the $\mathrm{CD}$ peak is heavily dependent on the sample rotation angles and much larger in amplitude due to strong LD artefacts (examples Figures $4 \mathrm{~b}$ and d, and SI). Although averaging the $\mathrm{N}$ phase spectra leaves a residual peak, LD contributions are too large and variable to sensibly determine chirality in this phase. Similar artefacts are also observed for $\mathrm{N}_{\mathrm{tb}}$ phases that are not homeotropically aligned (Figure S7, SI). As expected, no CD signal was observed in the isotropic phase. 

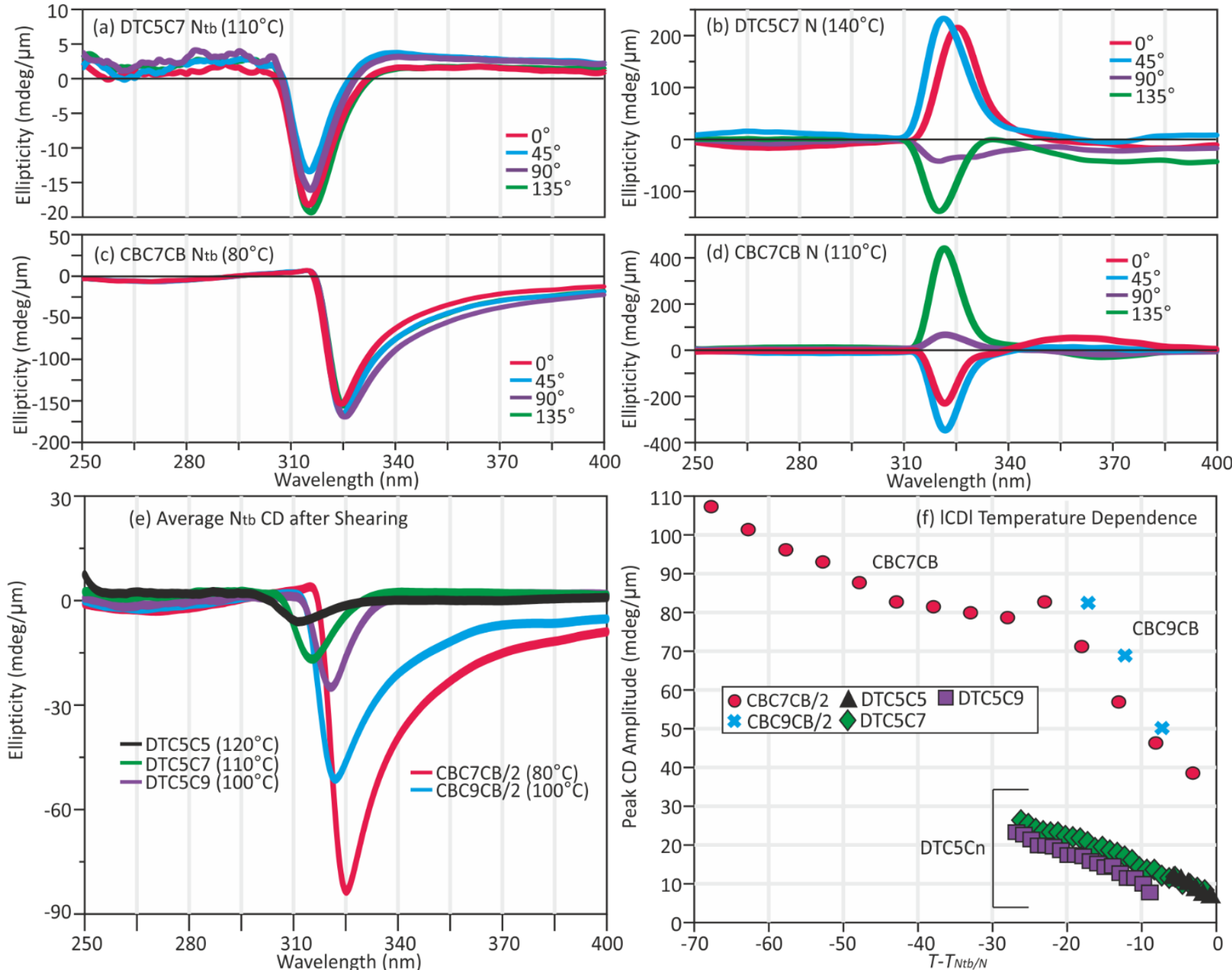

Figure 4 - (a) CD spectra of the homeotropically aligned (sheared) Ntb phase In DTC5C7, (b) N phase in DTC5C7, (c) Sheared $N_{\text {tb }}$ phase in $C B C 7 C B$ and (d) N phase in CBC7CB. (e) The orientational average CD spectra of each sample after shearing in $\mathrm{N}_{\text {tb }}$ phase. Note that $\mathrm{CBCnCB}$ spectra have been divided by 2 for display purposes. CBC9CB data (positive peak) have been inverted for easier comparison. (f) Temperature dependence of peak $C D$ amplitude. $T$-scale is relative to $\mathrm{N}_{\mathrm{tb}} / \mathrm{N}$ transition temperature.

The temperature dependence of $\mathrm{CD}$ was investigated in the $\mathrm{N}_{\mathrm{tb}}$ phase each sample. $\mathrm{CD}$ spectra were recorded every $5^{\circ} \mathrm{C}(\mathrm{CBCnCB})$ or every $1^{\circ} \mathrm{C}(\mathrm{DTC} 5 \mathrm{Cn})$. Measurements above $T_{\text {shear }}$ were made on heating and those below on cooling, however the time between temperature increments was around 10 minutes, giving samples time to equilibrate. Note that loss of homeotropic alignment at high temperature and isothermal crystallization at low temperature mean the workable $\mathrm{N}_{\mathrm{tb}}$ temperature range is smaller than suggested by DSC. The peak CD signal for each sample is plotted against $T$ $T_{\mathrm{Ntb} / \mathrm{N}}$ in Figure $4 \mathrm{f}$.

\section{Discussion}

In DTC5Cn compounds CD amplitude reduces linearly as the sample approaches the $\mathrm{N}$ phase. This shows that the phase becomes less twisted with increasing temperature. In a dimer mixture labelled "Se 45 ", containing 55\% DTC5C7 and 45\% of a selenium-labelled compound, it was shown by resonant X-ray scattering that the $\mathrm{N}_{\mathrm{tb}}$ pitch length increases towards the $\mathrm{N}$ phase. ${ }^{20}$ A lengthening pitch but reducing twisting power supports our previous hypothesis that the molecules straighten with 
increasing temperature, causing the helix to become longer, but less tightly coiled, as the $\mathrm{N}$ phase is approached. ${ }^{34}$ In DTC5C9, birefringence contributions became an issue at temperatures above $T-T_{\mathrm{Ntb} / \mathrm{N}}$ $\approx-7.5^{\circ} \mathrm{C}$, preventing reliable measurement of chirality. The presence of birefringence in this $T$-range was later confirmed using POM. The development of birefringence close to the $\mathrm{N}$ phase is consistent with the vanishingly small $\mathrm{N}_{\mathrm{tb}} / \mathrm{N}$ transition enthalpy $(60 \mathrm{~mJ} / \mathrm{g})^{34}$, allowing $\mathrm{N}$ phase defects to develop in the homeotropic alignment, or simply N-type fluctuations destabilizing the homeotropic alignment, which is not maintained in the $\mathrm{N}$ phase.

In $\mathrm{CBCnCB}$ samples, $\mathrm{CD}$ also reduces with increasing temperature. This is consistent with NMR findings where quadrupolar splitting, thought to arise from phase asymmetry, also reduces with increasing temperature. ${ }^{9,23,24}$ There is a significant $\mathrm{CD}$ gradient change around $T_{\text {shear }}\left(T-T_{\mathrm{Ntb} / \mathrm{N}}=-24^{\circ} \mathrm{C}\right)$ is observed (Figure 4f). Above this temperature $\mathrm{CD}$ drops rapidly as $T$ approaches the $\mathrm{N}_{\mathrm{tb}} / \mathrm{N}$ phase transition. In $\mathrm{CBC} 9 \mathrm{CB}$ isothermal crystallization occurs at $85^{\circ} \mathrm{C}$, preventing exploration of lower temperatures. The break in slope around the $T_{\text {shear }}$ could be an artefact due to sample thermal history.

While CD spectroscopy of all five compounds reported here shows global chirality in the $\mathrm{N}_{\mathrm{tb}}$ phase, further work is needed on other $\mathrm{N}_{\mathrm{tb}}$ forming dimers, trimers, oligomers, polymers as well as mixtures, to establish the universality of the phenomenon. The possibility of achieving homeotropic alignment, of the $\mathrm{N}_{\mathrm{tb}}$ phase for CD experiments, using LC cells of fixed thickness, could be explored in the future, but suitable UV transparent materials for electrodes and alignment layers, or new $\mathrm{N}_{\mathrm{tb}}$ compounds with chromophores in the visible region, will have to be used.

Finally a comment on shear alignment of the $\mathrm{N}_{\mathrm{tb}}$ phase. Ordinary nematic normally align parallel to the shear direction. This property is exploited widely, e.g. in the spinning of strong and stiff fibres of LC polymers (e.g. Kevlar or Vectra) from their nematic solutions or melts. In contrast, smectic LCs normally align with their layers parallel to the shear direction, which in the case of Sm-A means with the director perpendicular to the shearing plates. The fact that $\mathrm{N}_{\mathrm{tb}}$ aligns like a smectic rather than like a nematic is likely to be due to its helical structure preventing simple translational diffusion characteristic of an ordinary nematic and instead favouring the transverse motion facilitated by its short-range smectic-like order evidenced by the diffuse SAXS peaks in Figure 3b, in line with the results of dynamic light scattering studies. ${ }^{40}$

\section{Conclusions}

In conclusion we have shown unequivocally, by the key chiroptical method of circular dichroism, that the low temperature nematic phase possesses marcoscopic chirality in the two archetypal series of bimesognes exemplifying this phase. It is shown that arfefact-free CD spectroscopy can be used on such systems, provided the materials can be aligned homeotropically. As the temperature is increased towards the $\mathrm{N}_{\mathrm{tb}} \rightarrow \mathrm{N}$ transition, all five studied compounds show a decreasing $\mathrm{CD}$, suggesting that the helix gradually unwinds as $\mathrm{N}$ phase is approached. The experiment provides a direct proof of the twistbend rather than splay-bend nature of the low-temperature nematic.

\section{Materials and Methods}

POM: The optical textures of DTC5Cn and $\mathrm{CBCnCB}$ compounds were investigated using an Olympus BX50 Microscope fitted with a Mettler FP82 HT hotstage. Each compound was placed between two regular glass plates and heated to the isotropic phase. The samples were then cooled stepwise through their respective phase sequences between crossed polarizers. 
GIXS: Experiments were conducted on station BM28 (bending magnet) of the ESRF. Each sample was melted onto a silicon substrate and placed on the surface of a custom temperature controlled stage on a six circle goniometer. The beam size used was $200 \mu \mathrm{m}$ x $50 \mu \mathrm{m}$ and the sample to detector distance was $272 \mathrm{~mm}$. A helium-filled flight tube was positioned between the sample and a Mar165 CCD detector. The shearing process was conducted in the $\mathrm{N}_{\mathrm{tb}}$ phase by repeatedly sliding a second heated silicon substrate over the surface of the sample.

CD/UV-Vis: experiments were conducted on station B23 of the Diamond Light Source using a custom-made instrument featuring an ultra-high vacuum Olis DSM20 monochromator and a photomultiplier tube (detector) with an $\mathrm{MgF}_{2}$ window. The beam diameter was approximately $100 \mu \mathrm{m}$ at the sample position. Inside the optical chamber, each sample was held in a quartz glass sandwich cell, which rested on a rotatable Linkam hot stage. The incident beam ran perpendicular to the surfaces of the quartz plates. The samples were held isothermally in the $\mathrm{N}_{\mathrm{tb}}$ phase and sheared in situ, i.e. while resting on the rotatable hot-stage inside the optical chamber. CD and UV-Vis spectra were simultaneously recorded in the wavelength range of 250-400 nm and the intensity was integrated for 0.5 seconds in each $1 \mathrm{~nm}$ increment. The rotatable stage was used to measure the CD spectrum in various sample orientations (every $45^{\circ}$ ) so that, if present, the contributions of birefringence and linear dichroism could be identified and reduced by averaging. Four angular increments were investigated due to the optical equivalence of $0^{\circ}$ and $180^{\circ}$ etc. The film thickness in each case was estimated by absorption calibration from cells of known thickness (see SI).

\section{Conflicts of interest}

There are no conflicts to declare.

\section{References}

1. G. Ungar, J. L. Feijoo, A. Keller, R. Yourd, V. Percec. Simultaneous X-ray/DSC study of mesomorphism in polymers with a semiflexible mesogen, Macromolecules, 1990, 23, 34113416.

2. G. Ungar, V. Percec, M. Zuber. Liquid crystalline polyethers based on conformational isomerism. 20. Nematic-nematic transition in polyethers and copolyethers based on 1-(4hydroxyphenyl)2-(2-R-4-hydroxyphenyl)ethane with $\mathrm{R}=$ fluoro, chloro and methyl and flexible spacers containing an odd number of methylene units, Macromolecules, 1992, 25, 7580 .

3. W. D. Stevenson, J. An, X. B. Zeng, M. Xue, H.-X. Zou, Y.-S. Liu, G. Ungar. Twist-bend nematic phase in biphenylethane-based copolyethers, Soft Matter, 2018, 14, 3003-3011.

4. M. Sepelj, A. Lesac, U. Baumeister, S. Diele, D. W. Bruce, Z. Hamersak. Dimeric Salicylaldimine-Based Mesogens with Flexible Spacers: Parity-Dependent Mesomorphism, Chem. Mater., 2006, 18, 2050-2058.

5. D. Chen, J. H. Porada, J. B. Hooper, A. Klittnick, Y. Shen, M. R. Tuchband, E. Korblova, D. Bedrov, D. M. Walba, M. A. Glaser, J. E. Maclennan, N. A. Clark. Chiral heliconical ground state of nanoscale pitch in a nematic liquid crystal of achiral molecular dimers, Proc. Natl. Acad. Sci. U.S.A., 2013, 110, 15931-15936.

6. V. Borshch, Y.-K. Kim, J. Xiang, M. Gao, A. Jákli, V. P. Panov, J. K. Vij, C. T. Imrie, M. G. Tamba, G. H. Mehl, O. D. Lavrentovich. Nematic twist-bend phase with nanoscale modulation of molecular orientation, Nat. Commun., 2013, 4, 2635. 
7. Z. Zhang, V. P. Panov, M. Nagaraj, R. J. Mandle, J. W. Goodby, G. R. Luckhurst, J. C. Jones, H. F. Gleeson. Raman scattering studies of order parameters in liquid crystalline dimers exhibiting the nematic and twist-bend nematic phases, J. Mater. Chem. C, 2015, 3, 1000710016.

8. C. Meyer, G. R. Luckhurst, I. Dozov. The temperature dependence of the heliconical tilt angle in the twist-bend nematic phase of the odd dimer CB7CB, J. Mater. Chem. C, 2015, 3, 318328.

9. M. Cestari, S. Diez-Berart, D. A. Dunmur, A. Ferrarini, M. R. de la Fuente, D. J. B. Jackson, D. O. López, G. R. Luckhurst, M. A. Perez-Jubindo, R. M. Richardson, J. Salud, B. A. Timimi, and H. Zimmermann. Phase behavior and properties of the liquid-crystal dimer 1",7"-bis(4cyanobiphenyl-4'-yl) heptane: A twist-bend nematic liquid crystal, Phys. Rev. E, 2011, 84, 031704.

10. D. Chen, M. Nakata, R. Shao, M. R. Tuchband, M. Shuai, U. Baumeister, W. Weissflog, D. M. Walba, M. A. Glaser, J. E. Maclennan, N. A. Clarke. Twist-bend heliconical chiral nematic liquid crystal phase of an achiral rigid bent-core mesogen, Phys. Rev. E, 2014, 89, 022506.

11. Y. Wang, G. Singh, D. M. Agra-Kooijman, M. Gao, H. K. Bisoyi, C. M. Xue, M. R. Fisch, S. Kumar, Q. Li. Room temperature heliconical twist-bend nematic liquid crystal, CrystEngComm, 2015, 17, 2778-2782.

12. S. M. Jansze, A. Martínez-Felipe, J. D. M. Storey, A. T. M. Marcelis, C. T. Imrie. A twist-bend nematic phase driven by hydrogen bonding, Angew. Chem. Int. Ed., 2015, 54, 643-646.

13. R. J. Mandle, J. W. Goodby. A Liquid Crystalline Oligomer Exhibiting Nematic and TwistBend Nematic Mesophases, ChemPhysChem, 2016, 17, 967-970.

14. F. Simpson, R. J. Mandle, J. N. Moore, J. W. Goodby. Investigating the Cusp between the nano- and macro-sciences in supermolecular liquid-crystalline twist-bend nematogens, $J$. Mater. Chem. C, 2017, 5, 5102-5110.

15. Z. Parsouzi, G. Babakhanova, M. Rajabi, R. Saha, P.Gyawali, T. Turiv, C. Welch, Georg $H$. Mehl, J. Gleeson, A. Jákli, O. D. Lavrentovich'S.Sprunt. Pretransitional behavior of viscoelastic parameters at the nematic to twist-bend nematic phase transition in flexible n-mers, Phys. Chem. Chem. Phys., 2019, 21, 13078-13089.

16. R. Saha, Z. Parsouzi, G. Babakhanova, Z. Parsouzi, , M. Rajabi, R. Saha, P.Gyawali, C. Welch G. H. Mehl, J. Gleeson, Oleg D. Lavrentovich, S.Sprunt, A. Jákli. Oligomeric odd-even effect in liquid crystals, Materials Horizon, 2019, DOI: 10.1039/C9MH00428A; accepted, $12 / 06 / 2019$.

17. I. Dozov. On the spontaneous symmetry breaking in the mesophases of achiral banana-shaped molecules, Europhys. Lett., 2001, 56, 247-253.

18. A. G. Vanakaras, D. J. Photinos. Molecular dynamics simulations of nematic phases formed by cyano-biphenyl dimers, Liq. Crsyt., 2018, 45, 13-15, 2184-2196.

19. E. Gorecka, M. Salamonczyk. A. Zep, D. Pociecha, C. Welch Z. Ahmed, G. H. Mehl. Do the short helices exist in the nematic TB phase? Liq. Cryst., 2015, 42, 1-7.

20. W. D. Stevenson, Z. Ahmed, X. B. Zeng, C. Welch, G. Ungar, G. H. Mehl. Molecular organization in the twist-bend nematic phase by resonant X-ray scattering at the Se K-edge and by SAXS, WAXS and GIXRD, Phys. Chem. Chem. Phys., 2017, 19, 13449-13454. 
21. C. Zhu, M. R. Tuchband, A. Young, M. Shuai, A. Scarbrough, D. M. Walba, J. E. Maclennan, C. Wang, A. Hexemer, N. A. Clark. Resonant carbon K-edge soft X-ray scattering from latticefree heliconical molecular ordering: soft dilative elasticity of the twist-bend liquid crystal phase, Phys. Rev. Lett., 2016, 116, 147803.

22. M. Salamończyk, N. Vaupotič, D. Pociecha, C. Wang, C. Zhu, E. Gorecka, Structure of nanoscale-pitch helical phases: blue phase and twist-bend nematic phase resolved by resonant soft X-ray scattering, Soft Matter, 2017, 13, 6694-6699.

23. A. Hoffmann, A. G. Vanakaras, A. Kohlmeier, G. H. Mehl, D. J. Photinos. On the structure of the Nx phase of symmetric dimers: inferences from NMR, Soft Matter, 2015, 11, 850-855.

24. G. R. Luckhurst, B. A. Timimi, N. J. Wells, H. Zimmermann. On orientational order in nematic and twist-bend nematic phases: a $2 \mathrm{H}-\mathrm{NMR}$ study of binary mixtures of the odd dimer, 1",9"bis(4-cyanobiphenyl-4'-yl) nonane, (CB9CB), and the monomer, 4-pentyl-4'-cyanobiphenyl, (5CB-d2), Liq. Cryst., 2018, 45, 13-15, 1913-1928.

25. K. Yamada, Y. Takanishi, K. Ishikawa, H. Takezoe, A. Fakuda, M. A. Osipov. Sign inversion of liquid-crystal-induced circular dichroism observed in the smectic-A and chiral smectic-Calpha phases of binary mixture systems, Phys. Rev. E, 1997, 56 (1), R43-R46.

26. J. Thisayukta, H. Niwano, H.Takezoe, J. Watanabe. Effect of chiral dopant on a helical Sm1 phase of banana-shaped N-n-O-PIMB molecules, J. Mater. Chem., 2001, 11, 2717-2721.

27. H. Nagayama, S. Kumar Varshney, M. Goto, F. Araoka, K. Ishikawa, V. Prasad, H. Takezoe. Spontaneous Deracemization of Disc-like Molecules in the Columnar Phase, Angew. Chem. Int. Ed., 2010, 49, 445-448.

28. S.-W. Choi, S. Kang, Y. Takanishi, K. Ishikawa, J. Watanabe, H. Takezoe. Intrinsic chiral domains enantioselectively segregated from twisted nematic cells of bent-core mesogens, Chirality, 2007, 19, 250-254.

29. C. Roche, H.-J. Sun, M. Prendergast, P. Leowanawat, B. Partridge, P. A. Heiney, F. Araoka, R. Graf, H. W. Spiess, X.B. Zeng, G. Ungar, V. Percec. Homochiral columns constructed by chiral self-sorting during supramolecular helical organization of hat-shaped molecules, J. Am. Chem. Soc., 2014, 136, 7169-7185.

30. L. Cseh, X. Mang, X.B. Zeng, F. Liu, G. H. Mehl, G. Ungar, Helically twisted chiral arrays of gold nanoparticles coated with a cholesterol mesogen, J. Am. Chem. Soc., 2015, 137, 1273612739.

31. C. Roche, H-J Sun, P. Leowanawat, F. Araoka, B. E. Partridge, M. Peterca, D. A. Wilson, M. E. Prendergast, P. A. Heiney, R. Graf, H. W. Spiess, X. B. Zeng, G. Ungar, V. Percec. A supramolecular helix that disregards chirality, Nature Chem., 2016, 8, 80-89.

32. J. C. Sutherland, Measurement of Circular Dichroism and Related Spectroscopies with Conventional and Synchrotron Light Sources: Theory and Instrumentation, Chapter 2 in Modern Techniques for Circular Dichroism and Synchrotron Radiation Circular Dichroism Spectroscopy. B. A. Wallace and R. W. Janes Eds, IOS Press, 2009.

33. J. Altschuh, T. Karstenst, H.-G. Kuball. A cuvette and the technique for circular-dichroism measurements of molecules oriented in a liquid-crystal matrix, J. Phys. E: Sci. Instrum., 1981, 14, 43-44. 
34. W. D. Stevenson, H.-X. Zou, X.-B. Zeng, C. Welch, G. Ungar, G. H. Mehl. Dynamic calorimetry and XRD studies of the nematic and twist-bend nematic phase transitions in a series of dimers with increasing spacer length, Phys. Chem. Chem. Phys., 2018, 20, 25268.

35. V. P. Panov, V. K. Vij, G. H. Mehl. Twist-bend nematic phase in cyanobiphenyls and difluoroterphenyls bimesogens, Liq. Cryst., 2017, 44, 1, 147-159.

36. V. P. Panov, M. Nagaraj, J. K. Vij, Y. P. Panarin, A. Kohlmeier, M. G. Tamba, R. A. Lewis and G. H. Mehl. Spontaneous periodic deformations in nonchiral planar-aligned bimesogens with a nematic-nematic transition and a negative elastic constant, Phys. Rev. Lett., 2010, 105, 167801 .

37. A. de Vries. X-ray photographic studies of liquid crystals I. A cybotactic nematic phase, Mol. Cryst. Liq. Cryst., 1970, 10, 219.

38. V. P. Panov, R. Balachandran, M. Nagaraj, J. K. Vij, M. G. Tamba, A. Kohlmeier, G. H. Mehl. Microsecond linear optical response in the unusual nematic phase of achiral bimesogens, App. Phys. Lett., 2011, 99, 261903.

39. C. Meyer, G. R. Luckhurst, I. Dozov. Flexoelectrically driven electroclinic effect in the twistbend nematic phase of achiral molecules with bent shapes, Phys. Rev. Lett., 2013, 111, 067801.

40. Z. Parsouzi, Shokir A. Pardaev, C. Welch, Z. Ahmed, G. H. Mehl, A. R. Baldwin, J. T. Gleeson, O. D. Lavrentovich, D. W. Allender, J. V. Selinger, A. Jakli, S. Sprunt. Light scattering study of the "pseudo-layer" compression elastic constant in a twist-bend nematic liquid crystal, Phys. Chem. Chem Phys., 2016; 18, 31645-31652.

\section{Acknowledgments}

$\mathrm{XMaS}$ (BM28, ESRF) is a UK national facility supported by EPSRC. We are grateful to all the beamline team staff for their support. This work is supported by EPSRC (EP-K034308, EP/M015726, EP-P002250), and the National Natural Science Foundation of China (No. 21674099). 